\title{
Ensemble of rankers for efficient gene signature extraction in smoke exposure classification
}

\author{
Maurizio Giordano ${ }^{*}$, Kumar Parijat Tripathi and Mario Rosario Guarracino \\ From Bringing Maths to Life 2017 \\ Naples, Italy. 07-09 June 2017
}

\begin{abstract}
Background: System toxicology aims at understanding the mechanisms used by biological systems to respond to toxicants. Such understanding can be leveraged to assess the risk of chemicals, drugs, and consumer products in living organisms. In system toxicology, machine learning techniques and methodologies are applied to develop prediction models for classification of toxicant exposure of biological systems. Gene expression data (RNA/DNA microarray) are often used to develop such prediction models.

Results: The outcome of the present work is an experimental methodology to develop prediction models, based on robust gene signatures, for the classification of cigarette smoke exposure and cessation in humans. It is a result of the participation in the recent sbv IMPROVER SysTox Computational Challenge. By merging different gene selection techniques, we obtain robust gene signatures and we investigate prediction capabilities of different off-the-shelf machine learning techniques, such as artificial neural networks, linear models and support vector machines. We also predict six novel genes in our signature, and firmly believe these genes have to be further investigated as biomarkers for tobacco smoking exposure.

Conclusions: The proposed methodology provides gene signatures with top-ranked performances in the prediction of the investigated classification methods, as well as new discoveries in genetic signatures for bio-markers of the smoke exposure of humans.
\end{abstract}

Keywords: Toxicology, Gene signature, Smoking, Supervised learning, Feature selection

\section{Background}

System toxicology aims at understanding mechanisms, both at functional and genetic structural level, by which biological systems respond to toxicants. Such understanding can be leveraged to assess the risk of chemicals, drugs, and consumer products on living organisms. In particular, the identification of effective genomic biomarkers to aid prediction of toxicant/drug exposure levels in biological systems is an emerging research topic in system toxicology.

*Correspondence: maurizio.giordano@cnr.it

High Performance Computing and Networking Institute (ICAR), National Council of Research (CNR), Naples, Italy
The increasing interest in this field is motivated by the wide applicability of genomic biomarkers for both finding evidence of toxicity in drug therapies and monitoring therapeutic outcomes. Furthermore, in case of acute poisoning, it can be used to detect exposure degree to toxicants/drugs. Indeed, the exposure level evaluation by safety biomarkers may lead to the development of more efficient diagnostic tools for toxicodynamic monitoring like in case of patients receiving immunosuppressive therapy [1]. This research area is relevant in many different applications, as shown by the identification of genomic biomarkers for a wide variety of toxicants, including nephrotoxic agents [2], testicular toxicants [3], for keratinocyte proliferation in papilloma murine skin 
model [4], and smoke exposure [5-7]. Several works propose the use of transcriptome-based exposure response signatures, computed by processing gene expression data (RNA/DNA microarray), to develop toxicant exposure prediction models [8-10]. In most of these approaches, gene signatures are identified by differential expression, using statistical tests involving case and control populations. Due to inter-individual variations present in human populations, observed gene sets could result in not-robust signatures. Indeed, robust signatures should maintain high specificity and sensitivity across independent subject cohorts, laboratories, and nucleic acid extraction methods.

In the present work we propose a methodology, as well as an experimental pipeline, for finding gene signatures for tobacco smoke exposure characterization and prediction. Our approach integrates different gene selection mechanisms, whose results are studied and compared to extract gene signatures more robust than those produced by a single methodology. In particular, the considered gene selection methods are based on a regression method (LASSO-LARS), a recursive elimination by support vector machines (RFE-SVM), and a feature selection by an ensemble of decision trees (Extra-Trees). While recent works start employing machine learning techniques for gene selection [11-13], the novelty of this work is to employ and merge the results from different gene selection methods, which are not limited to statistical analysis ones.
The sbv IMPROVER project [14] is a collaborative effort led and funded by Philip Morris International Research and Development which focuses on the verification of methods and concepts in systems biology research within an industrial framework. sbv IMPROVER project has recently proposed the SysTox Computational Challenge [15] aiming at exploiting crowdsourcing as a pertinent approach to identify and verify chemical cigarette smoking exposure response markers from human whole blood gene expression data. The aim is to leverage these markers as a signature in computational models for predictive classification of new blood samples as part of the smoking exposed or non-exposed groups (see Fig. 1). In this application domain we investigated our methodology for gene expression data processing and selection as a machine learning problem of feature selection/reduction in a data space with high dimensionality (in the order of thousands of variables). In this context, we demonstrate how the blood gene signatures we found with our methodology have large overlaps with those found by other related works. In addition we identified new genes which are not mentioned in literature as possible biomarkers for tobacco smoke exposure. The functional annotation and terms enrichment analysis, together with toxicogenomics analysis (chemical-gene-disease-pathway association studies), showed that the expression levels of these new genes are affected by smoke exposure. In addition, based on our signatures we obtained higher performances in terms of area under precision-recall curve (AUPR) and matthews

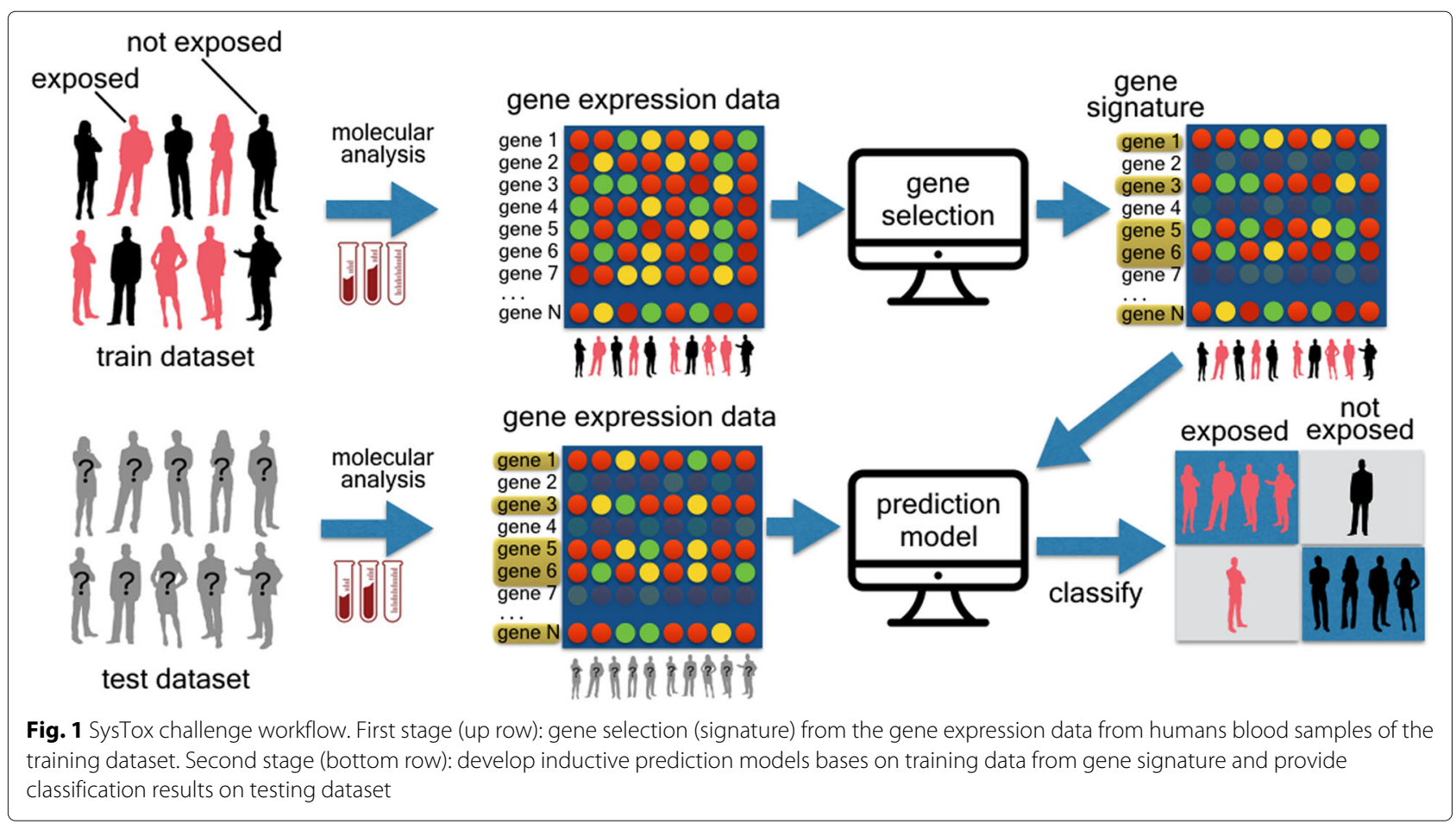


correlation coefficient (MCC) metrics by simply using a support vector machine (SVM) as a prediction model.

\section{Materials}

In the SysTox Computation Challenge $[15,16]$ participants were asked to develop models to classify subjects as smokers versus non-current smokers (SvsNCS), and then former smokers versus never smokers (FSvsNS), based on the information from whole blood gene expression data from humans (subchallenge 1), or humans and rodents (subchallenge 2). The current investigation focuses only on tasks referring to subchallenge 1 .

Figure 1 depicts the workflow of mandatory tasks the challengers were asked to follow. The workflow is the same as in the two classification problems proposed by the challenge. In the first stage of the challenge, a training dataset of gene expression data from human (or human/rodent) blood samples was made available for download to participants. The first task to be done was gene selection from whole blood gene expression data contained in the training dataset. The result of this task is a robust gene signature to be used to reduce training and testing data dimensions. Participants had also to develop inductive prediction models based on training data limited to the gene signatures they had previously identified. Inductive models are developed based only on training data. Classification on each test sample could be carried out only with the previously developed model, without retraining. Inductive models are different from transductive models in which training and testing datasets are processed together and used to retrain models prior to classification prediction. After all participants had submitted their results, in terms of both gene signatures and prediction models, the second stage of the challenge started: testing dataset of gene expression data from human (or human/rodent) blood samples were made available to participants. By using their proposed signatures and predictors, participants had to produce predictions (in terms of probabilities) on testing (unlabeled) samples.

After the competition closing, challenge organizers evaluated results submitted by participants only on a subset of testing samples which had been provided during the competition, the so called gold labels. Prediction models scores and rankings are reported on the sbv IMPROVER SysTox Challenge website.

Human blood sample data are organized in two datasets:

- H1 training dataset: a clinical case-control study conducted at the Queen Ann Street Medical Center (QASMC), London, UK and registered at ClinicalTrials.gov with the identifier NCT01780298

$[5,17]$. The QASMC study aimed at identifying biomarkers to discriminate smokers with chronic obstructive pulmonary disease (COPD) (i.e., cigarette smoke with a $\geq 10$ pack/year smoking history and COPD disease classified as GOLD Stage 1 or 2) from three groups of subjects which are matched by ethnicity, sex, and age (within 5 years) with the recruited COPD subjects: smokers (S), former smokers (FS), and never smokers (NS). All smoking subjects (S and FS) had a smoking history of at least 10 pack-years. FS quit smoking at least 1 year prior to sampling ( $\sim 78 \%$ of FS have stopped for more than 5 years). Patients included males (58\%) and females (42\%) aged between 40 and 70 years.

- H2 testing dataset: a transcriptomics dataset (BLDSMK-01) produced from PAXgene ${ }^{T M}$ blood samples obtained from a biobank repository (BioServe Biotechnologies Ltd., Beltsville, MD, USA) [5]. At the sampling time, the subjects were between 23 and 65 years of age. Subjects with a disease history and those taking prescription medications were excluded. Smokers (S) had smoked at least 10 cigarettes daily for at least three years. Former smokers (FS) quit smoking at least two years before the sampling and before cessation had smoked at least 10 cigarettes daily for at least three years. Smokers (S) and never smokers (NS) were matched by age and sex, while former smokers could not be properly matched due to the lower number of samples available for this group.

Sample data of H1 and H2 consist of DNA microarray experiments obtained with GeneChip Human Genome U133 Plus 2.0 Array and GeneChip Mouse Genome 430 2.0 Array (Affymetrix), on blood samples. Microarray data of both $\mathrm{H} 1$ and $\mathrm{H} 2$ are available in the ArrayExpress database [18], respectively under accession numbers E-MTAB-5278 and E-MTAB-5279. The distribution of training and testing labels and their categories are depicted in Fig. 2. For the human samples, 18604 gene expression data were provided.

\section{Methods \\ Gene selection}

The basic idea of our gene signature extraction approach is to identify an overlapping among the most discriminant genes we found out by applying three different feature selection techniques:

1 Feature selection by importances in forests of trees (Extra-Trees) [19]

2 Cross-validated Lasso, using the LARS algorithm [20]

3 Recursive Feature Elimination with SVM estimator [21]

Extra-Trees belong to the class of ensemble learning methods. They are based on bagging several instances of a black-box estimator (e.g. a decision tree) on random 


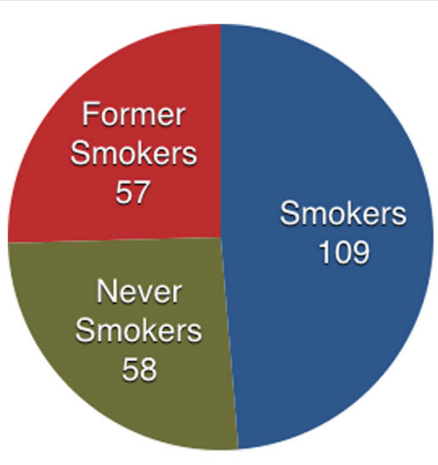

H1 training dataset (QASMC)

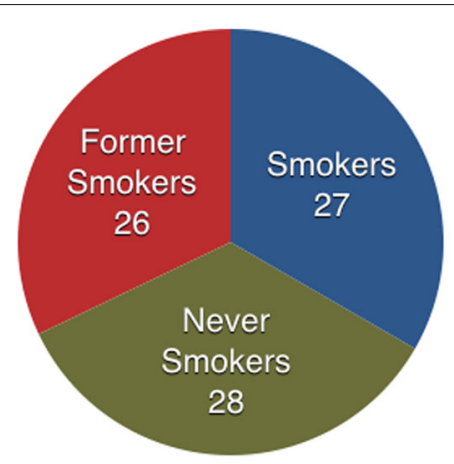

H2 testing dataset (BLD-SMK-01)

Fig. 2 SysTox challenge datasets. Distributions of training labels and testing (gold) labels into classes of subjects: smokers (treated group), former smokers (cessation group), and never smokers (control group)

subsets of the original training set and then combining their individual predictions to form a final prediction. Bagging estimators is a very simple way to improve with respect to a single model without making it necessary to adapt the underlying base algorithm. In many cases, bagging methods reduce overfitting as well as the variance of a base estimator. In this work we use the feature selection facility of the Extra-Trees implementation available in the Python Scikit-learn [22].

LASSO (Least Absolute Shrinkage and Selection Operator) is a regression method performing feature selection by regularization of regression parameters (e.g. constraining the sum of their absolute values). The computation of the LASSO solutions is a quadratic programming problem, and can be tackled by standard numerical analysis algorithms that estimate sparse coefficients. It is widely recognized that the Least Angle Regression procedure (LARS) is the better approach since it exploits the special structure of the LASSO problem, and it provides an efficient way to compute the solutions simultaneously for all values of the regularization parameter. In this work we use the LASSO method with LARS algorithm for feature selection. In the remaining of the paper we will refer to this feature selection method as LASSO-LARS. In particular we use its implementation available in the Python Scikit-learn library.

Recursive Feature Elimination with SVM (RFE-SVM) By starting with the complete set of features, RFE-SVM repeats the following three steps until no more features are left: 1) train a SVM model; 2) compute a ranking of features as the squares of the hyperplane coefficients of the SVM model; and 3) remove the features with the worst ranks. In this work we use the RFE-SVM implementation available in Weka Data Mining Software [23].

The three methods produce as outputs three lists of ranked genes in reversal order. Regardless of the ranking criteria (respectively as Decision Treed importance scores, LASSO coefficient estimates, and SVM hyperplane coefficients) the three lists of genes are cut-off to the first hundred of genes with higher ranks.

\section{Prediction models}

The focus of this work is on the data processing methodology to get a robust gene signature. The idea is that if the gene signature is biologically relevant, then classifiers will

Table 1 Prediction models

\begin{tabular}{|c|c|c|}
\hline Classifier & Acronym & Parameters \\
\hline Random forests & RF & $\begin{array}{l}\text { split=gini, max depth=none, } \text { min } \\
\text { samples leaf }=1 \text {, min samples } \\
\text { split=1, max features=auto, no. } \\
\text { estimators }=10\end{array}$ \\
\hline Gaussian Naive Bayes & GNB & none \\
\hline k-Nearest neighbors & kNN & $\begin{array}{l}\text { no.neighbors }=3 \text {, algorithm=auto, } \\
\text { metric }=\text { minkowski, } p=2, \\
\text { weights=uniform, leaf size }=30\end{array}$ \\
\hline MultiLayer perceptron & MLP & $\begin{array}{l}\text { activation=relu; algorithm=l-bfgs, } \\
\alpha=1 \text { e- } 05, \beta 1=0.9 \text {, beta } 2=0.999, \\
\epsilon=1 \text { e- } 08, \text { hidden layer sizes }=(100,)\end{array}$ \\
\hline Support vector classifier & SVC & $\begin{array}{l}\text { kernel=linear, } C=0.1 \text {, } \\
\text { tolerance }=0.001\end{array}$ \\
\hline Logistic regression & LR & $\begin{array}{l}\mathrm{C}=1.0 \text { max iter }=100 \text { penalty }=\mathrm{L} 2 \\
\text { tolerance }=0.0001, \text { multi class }=\text { OvR }\end{array}$ \\
\hline $\begin{array}{l}\text { Linear discriminant } \\
\text { analysis }\end{array}$ & LDA & solver $=S V D$, tolerance $=0.0001$ \\
\hline Gradient tree boosting & GTB & $\begin{array}{l}\text { loos }=\text { deviance, subsample }=1.0 \\
\text { learning rate }=0.1 \text {, min sample } \\
\text { split }=2 \text {, mean sample leaf }=1 \text {, } \max \\
\text { depth }=3 \text {, estimators }=100\end{array}$ \\
\hline $\begin{array}{l}\text { Extremely randomized } \\
\text { trees }\end{array}$ & ERT & $\begin{array}{l}\text { split=gini, max depth=No, } \min \\
\text { samples leaf }=1, \text { min samples } \\
\text { split=1, max features=auto, no. } \\
\text { estimators }=10\end{array}$ \\
\hline
\end{tabular}

The set of nine prediction models built by means of supervised learning on expression data (from $\mathrm{H} 1$ training dataset) of gene signatures 
Table 2 RFE-SVM SVSNCS signature

\begin{tabular}{llllllllll}
\hline AHRR & LRRN3 & SASH1 & CDKN1C & SEMA6B & RAD52 & FSTL1 & DSC2 & SYCE1L & TMEM163 \\
CRACR2B & MOG & ZP4 & KIT & P2RY6 & AK8 & PLA2G4C & MIR4697HG & SPAG6 & ZNF618 \\
CLEC10A & COL5A1 & B3GALT2 & TREM2 & TYR & MMP3 & LHX8 & KCNJ2-AS1 & ST6GALNAC1 & SCIN \\
SPRY2 & ADRA2A & GCNT3 & PTGFR & PACRG-AS1 & LINC00599 & NR4A1 & CHI3L1 & TPPP3 & SLC25A20 \\
NT5C1A & TCEB3B & BMP7 & FANK1 & TMTC1 & FGD5 & APCDD1L & GYS2 & TIMM8A & PID1 \\
SHISA6 & MYO1E & ADIRF-AS1 & CTTNBP2 & H19 & P2RY12 & DSTNP2 & MAGI2-AS3 & VSIG4 & NR4A2 \\
ICA1L & GFRA2 & GSE1 & NPIPB15 & ZFP64 & AFF3 & FOXC2 & CCR10 & ARHGAP32 & GPR15 \\
RRNAD1 & NOP9 & HYPM & PTGFRN & SLC25A27 & C3Orf65 & ZMYND12 & TM4SF4 & C6Orf10 & DUSP4 \\
FUCA1 & PALLD & ETNPPL & HMGCS2 & LMOD3 & EFNB1 & FABP4 & WNT2 & FAM187B & LINC01270 \\
PRKG2 & NMNAT2 & CYP4A11 & FAM19A2 & S1PR5 & LINC00544 & LRPAP1 & CTSV & LOC200772 & THBS2 \\
\hline
\end{tabular}

Gene signature obtained with Recursive Feature ith SVM in in smokers versus non-current smoker case study. Gene names in bold are also present in the signatures found by Extra-Trees and LASSO-LARS methods

provide statistically significant results. Therefore, in order to assess the quality and robustness of our gene selection method, on the basis of signatures produced by it, we built a large set of prediction models exploiting wellknown supervised learning techniques. We considered a set of nine classifiers, ranging from decision trees to support vector machines, from artificial neural networks to clustering and statistic methods. For the purpose, we used implementations of machine learning techniques available in the opensource Python Scikit-learn library [22]. The list of classifiers, their parameters setting and acronyms are reported in Table 1. All methods run in their default parameter configuration, since we were not interested in fine-tuning of each classifier.

\section{Biological and toxicological interpretation of gene signatures}

To understand the importance of gene signatures with respect to biological function and toxicological effects, we used Comparative Toxicogenomics Database (CTD) [24] and Transcriptator web-application [25] for the enrichment analysis of chemical association, diseases, pathways and gene ontology terms for our gene signatures. The CTD database is publicly available and provides knowledge about how environmental exposures affect human health. It contains both the curated and inferred information regarding chemical-gene/protein interactions, chemical-disease and gene-disease relationships. The functional gene ontology and pathway data related to genes are also included to study the possible mechanisms underlying environmentally influenced diseases. The curated information about gene-chemical interaction, gene-disease association and chemical-disease association is basically obtained through literature. Inferred relationships between gene-disease, gene-chemical and chemicaldisease association are established via CTD. For example in case of gene-disease-chemical association network, gene $\mathrm{A}$ is associated with disease $\mathrm{B}$ because gene $\mathrm{A}$ has a curated interaction with chemical $\mathrm{C}$, and chemical $\mathrm{C}$ has a curated association with disease $\mathrm{B}$. The database provides inference scores for all inferred relationships. These scores reflect the degree of similarity between CTD

Table 3 Extra-Trees SvsNCS signature

\begin{tabular}{llllllllll}
\hline LRRN3 & LINC00599 & P2RY6 & CDKN1C & GPR15 & AHRR & CTTNBP2 & DSC2 & CLEC10A & PF4 \\
RGL1 & SASH1 & FSTL1 & PTGFRN & C15orf54 & MCOLN3 & F2R & P2RY1 & GUCY1A3 & NRG1 \\
SEMA6B & ESAM & CR1L & PID1 & GP1BA & MAPK14 & PBX1 & GNAZ & GP6 & TMEM163 \\
RNASE1 & SLC44A1 & ASGR2 & GUCY1B3 & ZNF101 & LTBP1 & TRIP6 & SRRD & PRR5L & CYSTM1 \\
B3GALT2 & GRAP2 & ANKRD37 & MKNK1 & BEX2 & SV2B & FAXDC2 & ST6GALNAC1 & ICOS & NFIB \\
TRDC & SLPI & CDK2AP1 & IL4R & GPR20 & SH2D1B & TLR5 & VIL1 & ITGB5 & IGSF9B \\
CDR2 & BTBD11 & ELOVL7 & ARL3 & TUBB1 & BZRAP1 & ADAMDEC1 & C2Orf8 & COCH & LOC100506870 \\
LOC100130938 & CA2 & P2RY12 & SH3BGRL2 & PCSK6 & PRTFDC1 & SAMD14 & CYP4A11 & ASAP2 & H19 \\
LOC283194 & BLCAP & GORASP1 & TGM2 & SLC26A8 & ZAK & PARD3 & MB21D2 & GP9 & S100A12 \\
FANK1 & TNFSF4 & ZNF618 & FAM210B & MYBPC3 & SLC35G2 & ASIC3 & SLC6A4 & CNST & PAPSS2 \\
\hline
\end{tabular}

Gene signature obtained with feature selection of Extra-Trees in smokers versus non-current smoker case study. Gene names in bold are also present in the signatures found by RFE-SVM and LASSO-LARS methods 
Table 4 LASSO-LARS SvSNCS signature

\begin{tabular}{llllllllll}
\hline CDKN1C & GPR15 & LRRN3 & GPR63 & P2RY6 & SASH1 & CLEC10A & AHRR & GSE1 & ARHGAP32 \\
DSC2 & CRACR2B & PTGFR & LHX8 & FSTL1 & SYCE1L & APCDD1L & OTC & PID1 & PTGFRN \\
TMEM163 & CCR10 & P2RY12 & B3GALT2 & ST6GALNAC1 & RAD52 & TRDC & BCLAF1 & KNTC1 & CLSTN3 \\
ZNF536 & ACAP1 & DLGAP5 & IFT140 & LAPTM4A & MTSS1 & SETD1A & CCP110 & GPRASP1 & USP34 \\
SPCS2 & PHACTR2 & TM9SF4 & HDAC9 & SART3 & BMS1 & KIAA0232 & DOCK4 & TBC1D5 & CEP104 \\
PIEZO1 & PTDSS1 & VPRBP & SECISBP2L & SLK & FAM65B & KIAA0195 & SNPH & EIF4A3 & RAPGEF5 \\
RASSF2 & KIAA0101 & JADE3 & KIAA0247 & ZFYVE16 & KIAA0513 & LZTS3 & RIMS3 & SNX17 & MLEC \\
TOX & DHX38 & RAB11FIP3 & HDAC4 & FRMPD4 & KMT2B & TBKBP1 & STARD8 & ZSCAN12 & RNF144A \\
ATG13 & KIAA0586 & PCDHA9 & MATR3 & NOS1AP & ZNF646 & SDC3 & KIAA0430 & DZIP3 & SAFB2 \\
EIF5B & IPO13 & WSCD2 & SLC25A44 & CEP135 & KIAA0040 & TTI1 & PPIP5K1 & PHF14 & FAM53B \\
\hline
\end{tabular}

Gene signature obtained with Least Absolute Shrinkage and Selection Operator (with Least Angle Regression procedure) in smokers versus non-current smokers case study. Gene names in bold are also present in the signatures found by RFE-SVM and Extra-Trees methods

chemical-gene-disease association networks and a similar scale-free random network. A high score, suggests a stronger connectivity. We obtained the chemical-genedisease association information for all the gene signatures. Later we filter out genes only associated to "Tobacco smoke exposure" with inference score cutoff $\geq 50$. We obtained the disease association, pathways enrichment and gene ontology enrichment for gene signatures and carried out comparison between them through set analysis using Venn diagram.

\section{Results and discussion}

\section{Gene selection}

Each feature selection technique has been applied to the datasets, in both SvsNCS and FSvsNS classification problems, by setting a limit to the maximum number of selected genes (one hundred). For each problem the three sets found have been intersected to find a robust gene signature.

In the case of SvsNCS problem the results of the first hundred top-ranked genes by applying the three selection criteria are presented in Tables 2, 3 and 4. The three lists of genes show an overlap (the gene names in bold in the table) in the topmost positions. The set of 14 genes shared by all three lists form the resulting gene signature we propose for the SvsNCS case study. In Fig. 3 we have reported the boxplot of expression data in the training dataset of the 14-gene signature obtained with our approach.

In the case of FSvsNS problem, the results of the first hundred top-ranked genes by applying the three selection criteria are presented in Tables 5, 6 and 7. In this case a small overlapping exists between the three lists of genes produced by the three selection criteria. In particular, only 4 genes are shared (the gene names in bold in the table). The set of 4 genes shared by all three lists form the resulting gene signature we propose for the FSvsNS case study. In Fig. 4 we have reported the boxplot of expression data in the training dataset of the 4-gene signature produced by our approach. The experiments showed that by removing the gene LCMT1-AS2 we obtained a more robust gene signature.

\section{Signatures biological interpretation}

With respects to the SvsNCS problem, the lists of the first hundred of top-ranked genes are reported in Tables 2, 3 and 4 . As we may note, these gene lists share 14 genes which are associated to very high ranks in all of them.

To analyze these signatures, we obtained the genechemical association results from CTD database and we selected genes which interact with tobacco smoke pollution with higher inference score. Later, we carried out inferred gene-disease association, pathways and gene

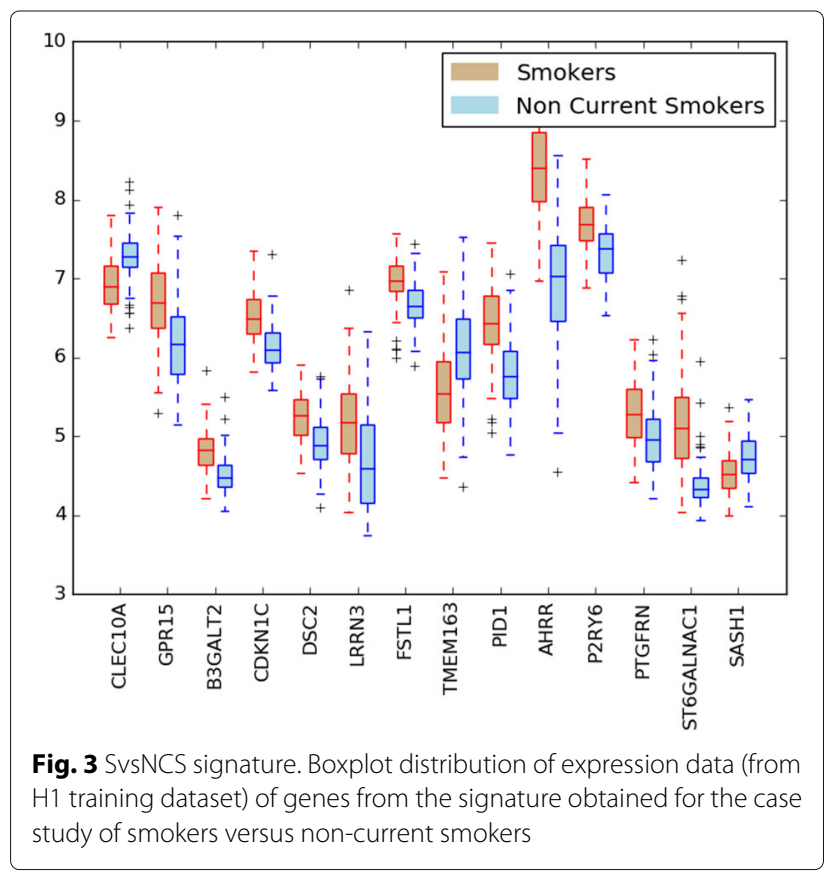


Table 5 RFE-SVM FSvSNS signature

\begin{tabular}{llllllllll}
\hline SLC38A3 & POU4F1 & HSD11B1 & GOLGA2P5 & IL17RD & CELF5 & ADAMTS14 & PTPN14 & MB21D2 & TBC1D29 \\
RRP12 & C4BPB & KRT73 & DCAF4 & ZNF280B & LOC648691 & DDX11 & TJP3 & LINC01097 & BCL2L12 \\
RAB42 & CLSPN & ADAM23 & CFD & TAS2R9 & CFAP46 & VSIG4 & GDF9 & SI & DOCK4-AS1 \\
SH3PXD2A-AS1 & CLUL1 & MMP1 & PLA2G2A & RTN3 & LY6G6D & ANKRD6 & IGSF9B & ZNF582-AS1 & C80rf88 \\
REG3A & ETV2 & NDST3 & C6orf99 & WNT5B & PAX4 & NNAT & HCG26 & SLC5A11 & TAAR3 \\
TTC22 & HAGHL & C17Orf78 & EDN2 & MTUS1 & PLCD4 & C1orf115 & PLEK & NS3BP & SLC34A2 \\
GGT5 & ZNF470 & SYN1 & SCD & MRAS & FOX11 & LCMT1-AS2 & HTN3 & SH3D19 & HIST1H4E \\
SHISA6 & MCOLN3 & LOC100507534 & SASH1 & APEX1 & C22Orf31 & RNF114 & SRRM4 & SCN2B & HMBOX1 \\
ATP6V1C2 & HSF4 & SLC17A5 & SEPT2 & TFAP4 & WWTR1 & FGF4 & SRCIN1 & SLC35F1 & SLC16A2 \\
TAS2R50 & PCAT19 & ADAMTS18 & TMEM31 & CAMK1G & SLC25A31 & SMR3B & SLC17A4 & XRCC6BP1 & PTPRB \\
\hline
\end{tabular}

Gene signature obtained with Recursive Feature Elimination with SVM in former smokers versus never smokers case study. Gene names in bold are also present in the signatures found by Extra-Trees and LASSO-LARS methods

Table 6 Extra-Trees FSvsNS signature

\begin{tabular}{llllllllll}
\hline MMP1 & PRR29 & APCS & HSD11B1 & DLK2 & NS3BP & CNTN2 & CLDN17 & CHGA & TMEM31 \\
MAPK10 & ZNF280B & C20orf85 & LDHD & CLUL1 & MAF & WFIKKN2 & CYP4B1 & NTRK3-AS1 & NKX6-1 \\
FAM221A & IFIT1 & SLC16A1 & HSD11B1L & LCMT1-AS2 & CLCN1 & IGSF9B & CENPU & ZNF652 & GPAM \\
ENTPD7 & FBXL19-AS1 & PRKCE & HCG26 & NLRP14 & B3GN77 & KLF14 & SLCO4A1 & SNCG & SLC34A2 \\
CEP76 & CXorf36 & ATF2 & STAU2-AS1 & SIGLEC11 & RWDD3 & ASB16 & FGB & HIST1H4H & ERN2 \\
CLRN1-AS1 & SLC50A1 & DOK4 & FASTKD1 & MB21D2 & HDAC1 & KIF2A & GMIP & CT83 & CYP2A13 \\
MED6 & CHDC2 & FGF13-AS1 & IFNA21 & DEPDC5 & CEP250 & MCM3AP & KRT75 & GLP1R & RAD51B \\
CFAP20 & TMEM184A & HOMEZ & LINC00922 & CRP & MAST1 & CBL & SDF4 & KRT19 & CELF5 \\
CDCA8 & ACTL8 & MRPS12 & ACER1 & SYCE3 & AP4E1 & TYK2 & LOC283914 & SLC12A1 & SCN2A \\
PLAC4 & OXCT1 & ABCA11P & GLB1 & TCEAL7 & LRRC32 & BHLHE22 & LINC01012 & TBK1 & TMEM225
\end{tabular}

Gene signature obtained with feature selection of Extra-Trees in former smokers versus never smokers case study. Gene names in bold are also present in the signatures found by RFE-SVM and LASSO-LARS methods

Table 7 LASSO-LARS FSVSNS signature

\begin{tabular}{llllllllll}
\hline POU4F1 & PTPRB & CLUL1 & SLC38A3 & PTPN14 & GDF9 & LCMT1-AS2 & C4BPB & LINC00901 & HSD11B1 \\
HSF4 & ADAMTS18 & SEPT2 & LOC648691 & EDN2 & LINC00319 & DOCK4-AS1 & TMEM246 & PBK & LINC00964 \\
SLC7A11 & IL17RD & TBC1D29 & PTPN3 & NS3BP & KIAA0513 & KIAA0586 & IFT140 & LAPTM4A & RNF144A \\
MATR3 & RIMS3 & SETD1A & CCP110 & GPRASP1 & USP34 & SNX17 & DHX38 & KNTC1 & HDAC9 \\
PIEZO1 & SART3 & DOCK4 & CEP104 & VPRBP & SECISBP2L & RAB11FIP3 & ZNF646 & TMEM63A & UTP14C \\
SEMA3E & NOS1AP & GPRIN2 & ARHGAP32 & ACAP1 & ZFYVE16 & PCDHA9 & KIAA0247 & LZTS3 & MLEC \\
TOX & HDAC4 & FRMPD4 & JADE3 & KMT2B & TBKBP1 & KIAA0101 & STARD8 & ZSCAN12 & SNPH \\
ZNF536 & FAM65B & RASSF2 & RAPGEF5 & SLK & KIAA0195 & BCLAF1 & EIF4A3 & ATG13 & TM9SF4 \\
CLSTN3 & KIAA0232 & TBC1D5 & PHACTR2 & KIAA0226 & ADAMTSL2 & KIAA0430 & MDC1 & IQCB1 & ZNF516 \\
PDE4DIP & CEP135 & LPIN2 & DZIP3 & TTLL4 & SAFB2 & EIF5B & IPO13 & WSCD2 & SDC3 \\
\hline
\end{tabular}

Gene signature obtained with Least Absolute Shrinkage and Selection Operator (with Least Angle Regression procedure) in former smokers versus never smokers case study. Gene names in bold are also present in the signatures found by RFE-SVM and Extra-Trees methods 


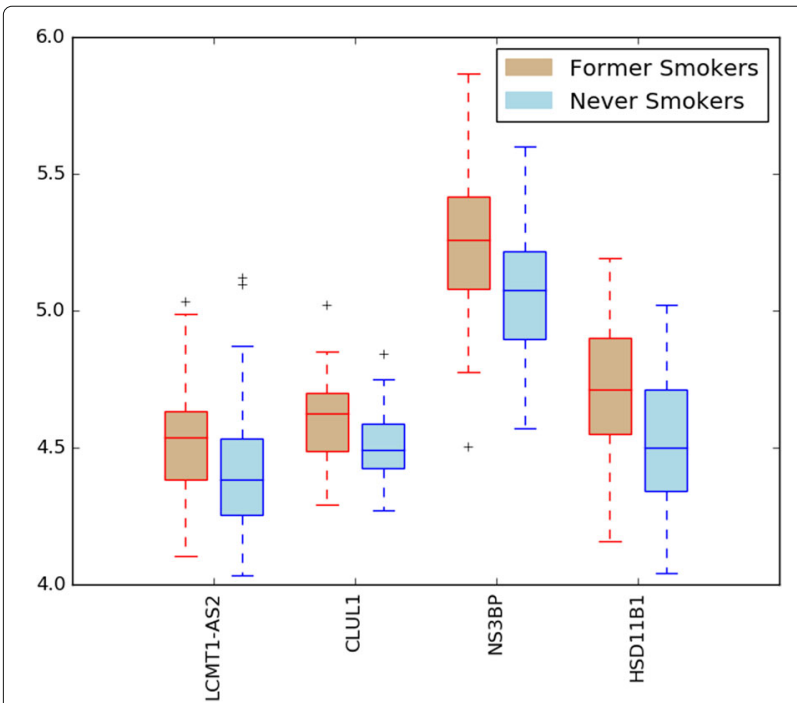

Fig. 4 FSvsNS signature. Boxplot distribution of expression data (from $\mathrm{H} 1$ training dataset) of genes from the signature obtained for the case study of former smokers versus never smokers

ontology enrichments analysis. The results are provided in the supplementary tables reported, in the 'Additional files' section, from 'Additional files 1, 2 and 3. The comparative analysis of disease association, pathway and gene ontology terms enrichment of the signatures obtained with the three gene selection techniques (Extra-Trees, LASSOLARS and RFE-SVM), provide a clear and robust picture of the signature associated with smoking effects. From our analysis (Fig. 5), we infer that though the overall overlap between the gene signatures from these methods is small, yet the gene signatures from the three methods shares a good amount of gene-disease association and most of these genes are involved in the same diseases.

We also observed that the diseases associated to these genes are respiratory tract, pregnancy complications, cardio-vascular, neoplasm, fetal disorder, congenital abnormalities, endocrine system diseases. Similarly, these genes share 74 common pathways, and some of these pathways (cell cycle, chemokine receptors bind chemokines, cytokine signaling in immune system, cytokine-cytokine receptor interaction, mitotic G1-G1/S phases, platelet activation, signaling and aggregation, post-translational protein modification, PPARA activates gene expression, Rap1 signaling pathway and Ras signaling pathway) are known to be involved in cancer progression.

The gene ontology enrichment and comparative analysis also suggest that most of these genes are involved in protein binding, membrane, localization, ion binding, regulation of biological process and signal transduction. In the light of these results, we deduce that the three gene signatures produced by our selection criteria, with respect to the smokers versus non-current smokers case

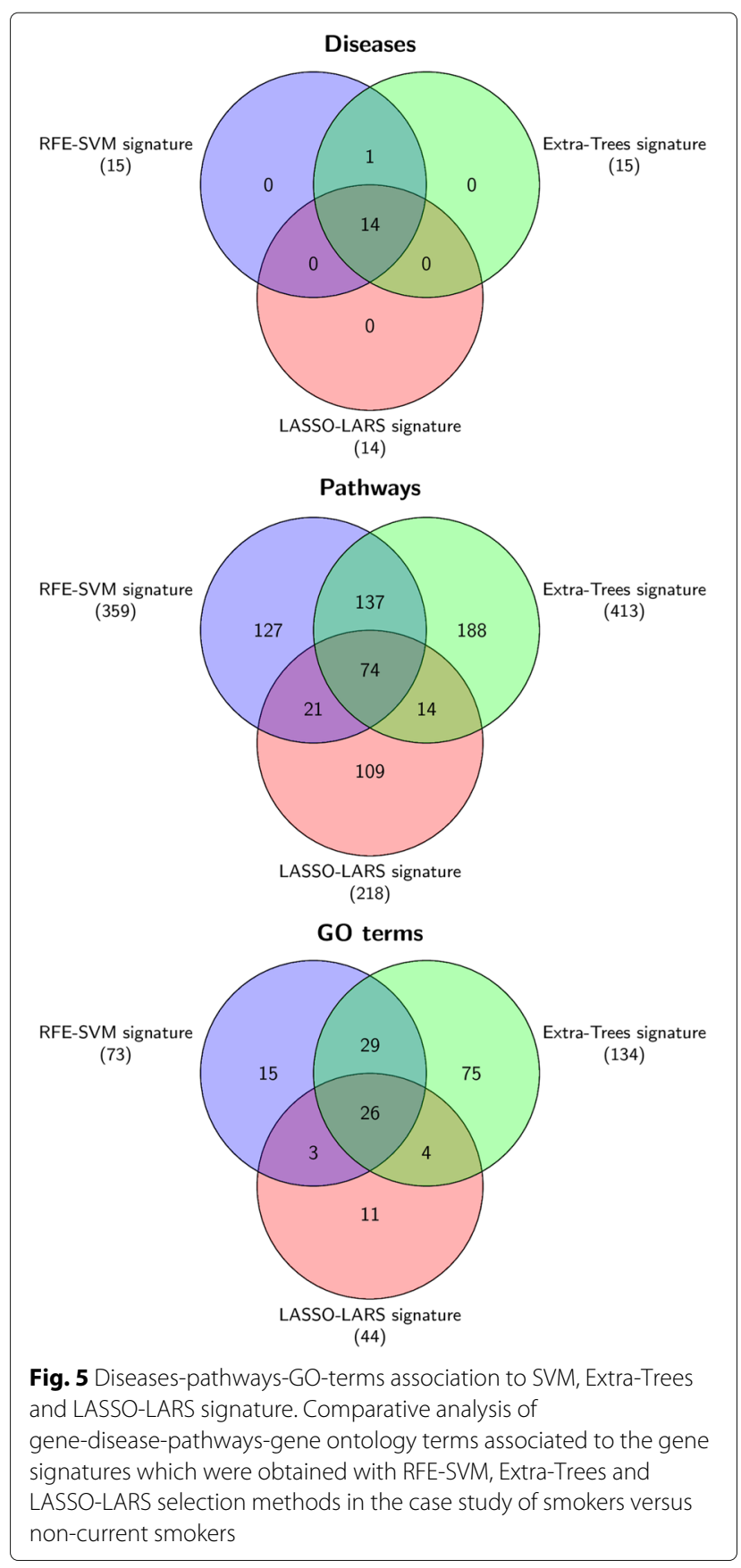

study, although different still share the same biological and toxicological characteristics. The overlap analysis among the three methods reported more stronger gene signature. We selected the genes common to all three methods and carried out the enrichment analysis.

The enrichment analysis of the gene signature we identified for the SvsNCS problem shows that all 14 genes are enriched (see Table 8) in biological processes, such as cellular response to chemical stimulus, and in molecular 
Table 8 SvsNCS signature biological interpretation

\begin{tabular}{lll}
\hline Gene name & Gene description & Chemical interaction \\
\hline CLEC10A & C-type lectin domain containing 10A & Benzo(a)pyrene \\
GPR15 & G protein-coupled receptor 15 & Tobacco Smoke Pollution \\
B3GALT2 & beta-1,3-galactosyltransferase 2 & Tobacco Smoke Pollution, Tretinoin, Valproic Acid, Vehicle Emissions \\
CDKN1C & cyclin-dependent kinase inhibitor 1C (p57, Kip2) & Tetrachlorodibenzodioxin, tert-Butylhydroperoxide, Valproic Acid \\
DSC2 & desmocollin 2 & Tetrachlorodibenzodioxin, Valproic Acid \\
LRRN3 & leucine rich repeat neuronal 3 & Tobacco Smoke Pollution \\
AHRR & aryl-hydrocarbon receptor repressor; programmed cell death 6 & Benzo(a)pyrene \\
TMEM163 & transmembrane protein 163 & Valproic Acid, Benzo(a)pyrene \\
PID1 & phosphotyrosine interaction domain containing 1 & Valproic Acid, Benzo(a)pyrene \\
FSTL1 & follistatin-like 1 & Methylnitronitrosoguanidine co-treated with Cadmium Chloride \\
P2RY6 & pyrimidinergic receptor P2Y, G-protein coupled, 6 & Benzo(a)pyrene \\
PTGFRN & prostaglandin F2 receptor inhibitor & Benzo(a)pyrene, Tetrachlorodibenzodioxin, Valproic Acid \\
ST6GALNAC1 & ST6 N-acetylgalactosaminide alpha-2,6-sialyltransferase 1 & Acetaminophen, Clofibrate, Phenylmercuric Acetate \\
SASH1 & SAM and SH3 domain containing 1 & Benzo(a)pyrene \\
\hline Enid
\end{tabular}

Enrichment analysis of the proposed gene signature in the smokers versus non-current smokers case study

functions, such as protein binding, ion binding, molecular transducer activity.

It is worth to notice that 4 genes from the proposed gene signature were already known in literature as biomarkers for cigarette smoke exposure. Indeed, genes LRRN3, SASH1, TNFRSF17, CDKN1C have been studied in [5], while LRRN3 gene was already known as biomarker in [26]. These genes were also found as biomarkers by the three winning teams participating in the SysTox Computational Challenge. Moreover these genes occupy the first positions in all the signatures that we identified. This is a further confirmation that our gene ranking criteria are in agreement with other approaches published in literature.

Similarly, we obtained the gene signatures for FSvsNS case study, by applying RFE-SVM, Extra-Trees and LASSO-LARS selection methods. The gene signatures are provided in Tables 5, 6 and 7 and they share only four genes.

In case of former smoker versus never smokers study, the enrichment analysis of the found gene signature shows that three genes which are included in our signatures (ADAMTS14, SLC38A3, HSD11B1), are known to contain SNPs or somatic mutations and differential expressed in lung/bladder cancers. The toxicogenomics gene-chemical-disease association study and the resulting biological and toxicogenomics data are provided in the supplementary tables reported, in the 'Additional file' section, from 'Additional files 4, 5, 6.

Table 9 shows the overlapping matrix of the gene signature resulting from our method with genes signatures produced by Philip Morris International (PMI) and by the three winning teams of the challenge (T264, T225 and T259) [27]. As we can see, in the overlap matrix our signature shares 8 out of 14 genes with the three teams (CLEC10A, GPR15, CDKN1C, LRRN3, AHRR, PID1, P2RY6, and SASH1). The remaining 6 genes (B3GALT2, DSC2, TMEM163, FSTL1, PTGFRN and ST6GALNAC1)

Table 9 Signature overlaps among methods

\begin{tabular}{|c|c|c|c|c|c|}
\hline Gene & Our & PMI & T264 & T225 & T259 \\
\hline CLEC10A & $\checkmark$ & & $\checkmark$ & $\checkmark$ & $\checkmark$ \\
\hline GPR15 & $\checkmark$ & & $\checkmark$ & $\checkmark$ & $\checkmark$ \\
\hline B3GALT2 & $\checkmark$ & & & & \\
\hline CDKN1C & $\checkmark$ & $\checkmark$ & $\checkmark$ & $\checkmark$ & $\checkmark$ \\
\hline DSC2 & $\checkmark$ & & & & \\
\hline LRRN3 & $\checkmark$ & $\checkmark$ & $\checkmark$ & $\checkmark$ & $\checkmark$ \\
\hline AHRR & $\checkmark$ & & $\checkmark$ & $\checkmark$ & $\checkmark$ \\
\hline TMEM163 & $\checkmark$ & & & & \\
\hline PID1 & $\checkmark$ & & $\checkmark$ & $\checkmark$ & $\checkmark$ \\
\hline FSTL1 & $\checkmark$ & & & & \\
\hline P2RY6 & $\checkmark$ & & $\checkmark$ & $\checkmark$ & $\checkmark$ \\
\hline PTGFRN & $\checkmark$ & & & & \\
\hline ST6GALNAC1 & $\checkmark$ & & & & \\
\hline SASH1 & $\checkmark$ & $\checkmark$ & $\checkmark$ & $\checkmark$ & $\checkmark$ \\
\hline RGL1 & & $\checkmark$ & & $\checkmark$ & $\checkmark$ \\
\hline SEMA6B & & & $\checkmark$ & $\checkmark$ & $\checkmark$ \\
\hline CTTNBP2 & & & $\checkmark$ & $\checkmark$ & \\
\hline$F 2 R$ & & & $\checkmark$ & $\checkmark$ & \\
\hline
\end{tabular}

Overlap matrix of the proposed gene signature with those produced by PMI and by the three winning teams of the SysTox Computational Challenge (for the smokers versus non-current smokers case study) 
were neither found by PMI nor by the winning teams. In the remaining of the document we will refer to the set of 8 genes shared by the three winning teams of the challenge as the common gene signature, while the set of 6 genes proposed only by us will be referred as specific gene signature. The completed set of 14 genes resulting from our method is referred as total gene signature.

We focused on these genes and carried out genechemical-pathways association studies using CTD database. The results are showed in Figs. 6 and 7 and in the supplementary tables reported, in the 'Additional files' section, from 'Additional files 7, 8, 9, 10, and 11'. Interestingly, we observe in Fig. 6 that the common gene signature has stronger affinity for smoke, tobacco smoke and Benzo(a)pyrene, the later being a constituent of cigarette smoke. By including in the analysis the 6 genes found only by us, we observe in Fig. 7 that the total gene signature still shows a stronger affinity for smoke and tobacco smoke.

We also determined the disease association for these 14 genes with inference score greater than threshold $(\geq 50)$ with respect to respiratory tract disease and respiratory insufficiency. Both these diseases of respiratory

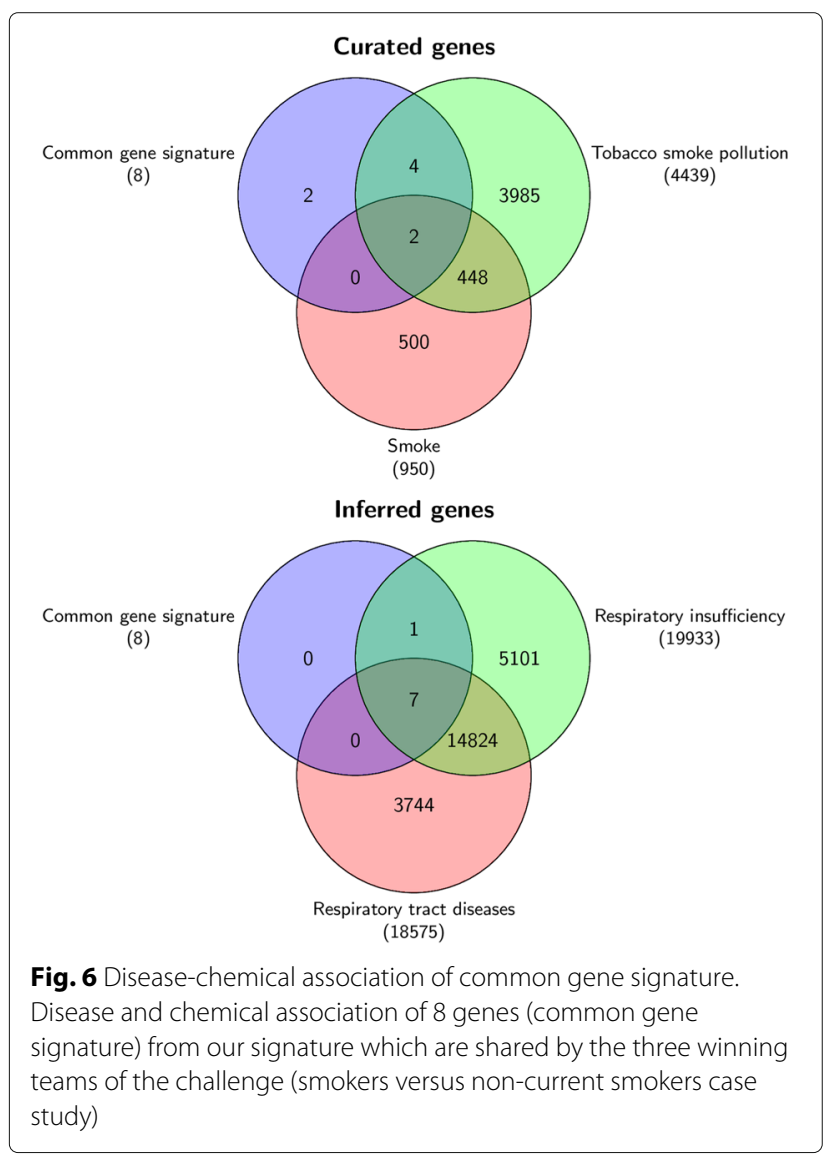

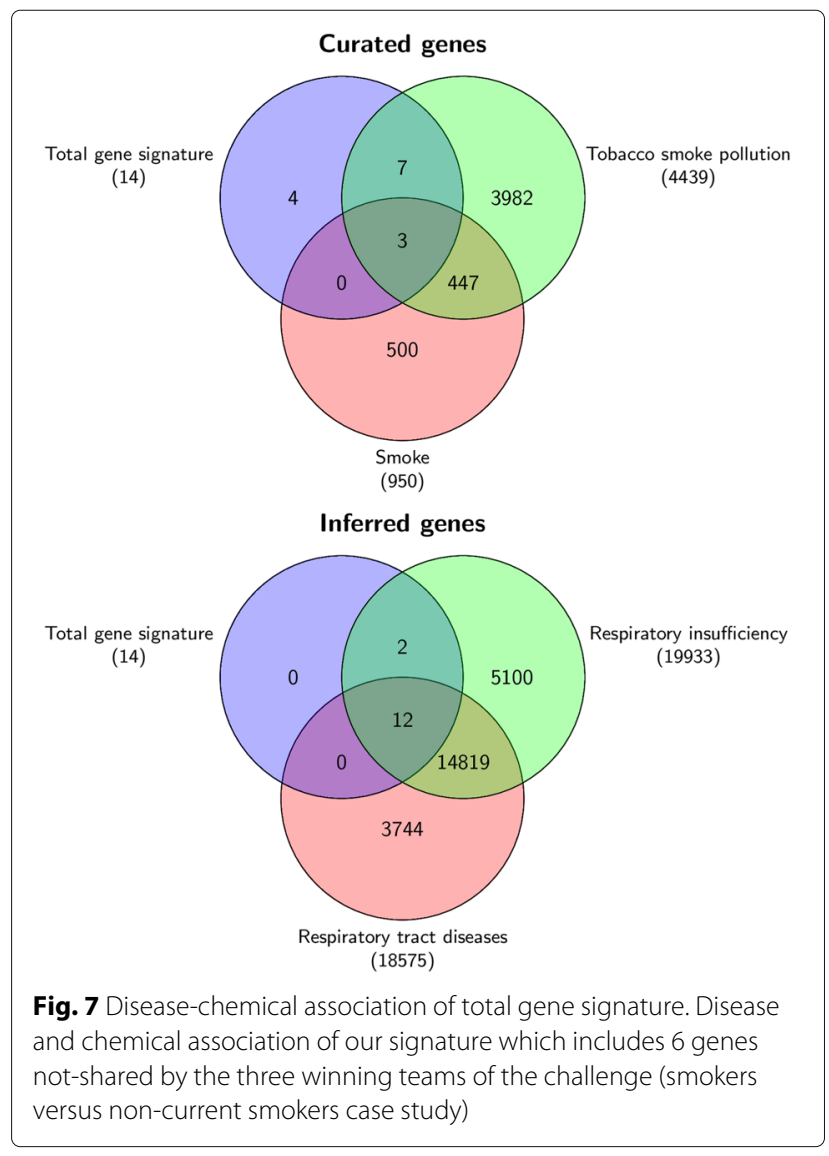

tract are well characterized in literature as a negative result of tobacco smoking.

We also carried out the pathways enrichment analysis for both the common gene signature and the specific gene signature in the case study of smokers versus non-current smokers. Biological and toxicogenomics analysis suggest that these 6 genes specific to our analysis are also very

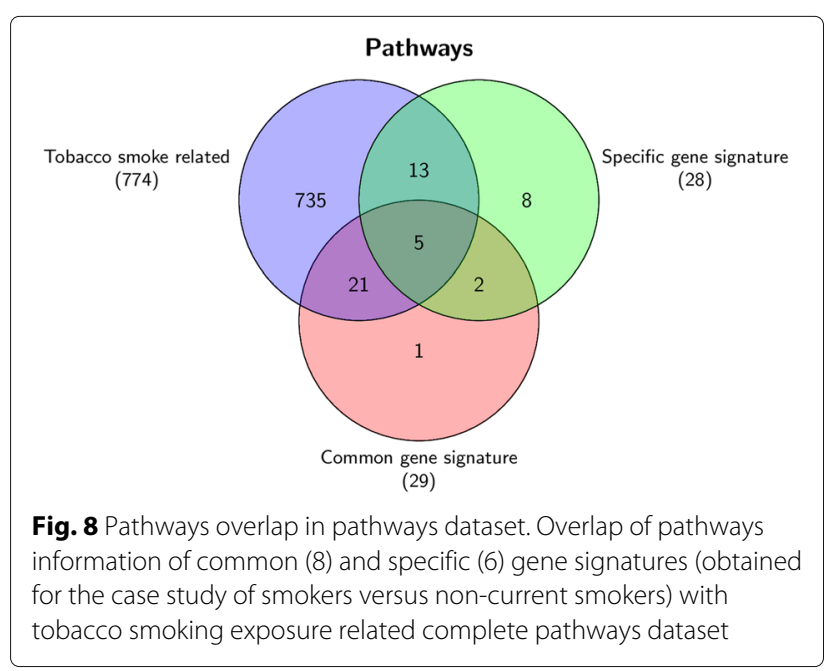


Table 10 FSvsNS signature biological interpretation

\begin{tabular}{lll}
\hline Gene name & Gene description & Chemical interaction \\
\hline CLUL1 & clusterin like 1 & Valproic Acid, bisphenol A \\
NS3BP & NS3 binding protein & Not Available \\
HSD11B1 & $\begin{array}{l}\text { hydroxysteroid 11-beta } \\
\text { dehydrogenase 1 }\end{array}$ & $\begin{array}{l}\text { Hydrocortisone, bisphenol A, } \\
\text { Tetrachlorodibenzodioxin }\end{array}$ \\
\hline
\end{tabular}

Enrichment analysis of the proposed gene signature in the former smokers versus never smokers case study

interesting with respect to smoking and could be further investigated as potential biomarkers for tobacco smoking exposure.

On comparing the enriched pathways in both common and specific gene signature with respect to the whole set of pathways associated with tobacco smoking, we determined the significant overlapped pathways for these 14 genes. Some of the main pathways are Class A/1 (rhodopsin-like receptors), GPCR downstream signaling, GPCR ligand binding, signal transduction and signaling by GPCR. The results are shown in Fig. 8. Out of 28 enriched pathways in specific gene signatures and 29 pathways in common gene signature, 18 and 26 pathways in both the signatures sets are effected by tobacco smoke. Most of these tobacco smoking associated pathways are involved in biological pathways such as cell signaling, platelet activation signaling and aggregation, post-translational protein modification, signaling by BMP, developmental biology, cell cycle, mitotic cyclin D associated events in G1, fatty acid, triacylglycerol and ketone body metabolism, G alpha (q) signalling events, innate immune system, metabolism, metabolism of lipids and lipoproteins and mitotic G1-G1/S phases. All these pathways are associated with the proper functioning of the cell.

The tabular results of pathways information associated with common and specific gene signature as well as the overlap analysis with tobacco smoking is provided in the 'Additional file 11'. Biological interpretation of these gene signatures using information from CTD database helps in the strengthening of our prediction model. More interestingly, we obtained a greater number of genes in our signature for smoker versus non-current smokers case study. The 6 genes which are not reported by other participants of the challenge, but suggested by our method, are also interesting and share the same biological and toxicological properties as the other genes of the signatures shared by the other participants. By taking into account these additional genes in our prediction model, we do have better chance to characterize smokers versus noncurrent smokers and surely this help in strengthening our prediction models over those proposed by the challengers.

With regards to the former smoker versus never smokers classification problem, we compared the gene signatures from the three selection methods and extracted three overlapping genes: CLUL1, NS3BP and HSD11B1. Biological and toxicological analysis of these three genes (see Table 10) suggests their chemical associations with valproic acid and tetrachlorodibenzodioxin. The later chemical is usually formed as a side product in organic synthesis and burning of organic materials and is a carcinogenic in nature. CLUL1 is involved in "Prenatal Exposure Delayed Effects" due to its chemical interactions with tetrachlorodibenzodioxin and bisphenol A. HSD11B1 is also involved in "Prenatal Exposure Delayed Effects" and it is also known to have chemical interactions with tetrachlorodibenzodioxin and bisphenol A.

\section{Prediction models}

Once the datasets for both SvsNCS and FSvsNS classification problems were reduced in such a way to contain only expression data of genes beloning to our signatures, we started a set of experiments with different classification methods. For the experiments we chose a subset of classifiers available in the Python Scikit-learn package. The list of classifiers, their parameters settings and acronyms are reported in Table 1 .

For both classification problems, we trained the classifiers on the $\mathrm{H} 1$ training dataset shrunk to the signature data. This supervised training procedure yielded to the construction of inductive prediction models for the two case studies. Later, the built models were used to classify (gold) samples from the $\mathrm{H} 2$ testing dataset, which of course had been previously reduced to the signature data.

With respect to the smokers versus non-current smokers classification problem, the prediction results of the nine selected classifier, in terms of AUPR and MCC scores, are summarized in Table 11 . The table reports also the scores obtained by the three winners of the challenge (T264, T225 and T259) for comparison. As we can see, the SVC classifier provided the best prediction performance (in both AUPR and MCC metric).

Table 11 Performance of classifiers using SvsNCS signature

\begin{tabular}{|c|c|c|c|c|c|c|c|c|c|c|c|c|}
\hline & RF & GNB & kNN & MLP & SVC & LR & LDA & GTB & ERT & T264 & T225 & T259 \\
\hline AUPR & 0.961 & 0.938 & 0.9140 & 0.9043 & 0.9746 & 0.9537 & 0.9484 & 0.9650 & 0.9580 & 0.96 & 0.97 & 0.95 \\
\hline MCC & 0.9012 & 0.8766 & 0.8025 & 0.8272 & 0.9259 & 0.8148 & 0.8765 & 0.9136 & 0.8642 & 0.90 & 0.77 & 0.79 \\
\hline
\end{tabular}

Performance measures, in terms of AUPR and MCC scores, of nine classifiers using the signature obtained for the case study of smokers versus non-current smokers. Results are compared to the scores obtained by winners of SysTox Computational Challenge. Best results in boldface 
Table 12 Performance of classifiers using FSvsNS signature

\begin{tabular}{|c|c|c|c|c|c|c|c|c|c|c|c|c|}
\hline & $\mathrm{RF}$ & GNB & kNN & MLP & SVC & LR & LDA & GTB & ERT & T264 & T225 & T259 \\
\hline AUPR & 0.6366 & 0.6357 & 0.6594 & 0.6710 & 0.7321 & 0.7024 & 0.6581 & 0.5528 & 0.6774 & 0.58 & 0.50 & 0.47 \\
\hline MCC & 0.0845 & 0.1092 & 0.1310 & 0.0307 & 0.2883 & 0.2318 & 0.1472 & -0.0644 & 0.1092 & 0.07 & 0.02 & -0.0 \\
\hline
\end{tabular}

Performance measures, in terms of AUPR and MCC scores, of nine classifiers using the signature obtained for the case study of former smokers versus never smokers. Results are compared to the scores obtained by winners of SysTox Computational Challenge. Best results in boldface

With respect to the former smokers versus never smokers classification problem, the AUPR and MCC scores of the selected classifiers are summarized in Table 12. As before, the table compares our results to the scores obtained by the three winners of the challenge. In this second case study, our results are more impressive, since the prediction scores are far better than those obtained by the other challengers.

\section{Conclusions}

The focus of this work is our contribution to the crowdsourcing initiative, namely the SysTox Computational Challenge, proposed by sbv IMPROVER project. The challenge initiative aims at identifying by crowdsourcing chemical cigarette smoking exposure biomarkers from human whole blood gene expression data.

In this context, this work proposed a methodology, as well as an experimental pipeline, to extract robust gene signatures from whole blood gene expression data. In addition, this work showed how to build predictive models based on robust gene signatures. Our models discriminate smokers from non-current smokers, as well as former smokers from never smokers subjects. In our computational approach we crossed three very different gene selection techniques to obtain robust gene signatures. Later, in order to assess the quality and robustness of the found gene signatures, we build, on the basis of expression data of selected genes of our signatures, nine prediction models implemented with different supervised machine learning techniques.

With regards to the SvsNCS classification problem we obtained high scores for the majority of the explored learning techniques, with AUPR and MCC scores comparable to (even better than) those obtained by the SysTox Challenge winners. Surprisingly, for what concerns the FSvsNS classification problem, the prediction models build on the basis of the found signatures performed far better than those proposed by the challenge winners.

The results obtained by our computational approach are strengthened by the functional annotation terms enrichment analysis, as well as by the toxicogenomics analysis (chemical-gene-disease-pathway association studies) for both the SvsNCS and FSvsNS gene signature. In case of SvsNCS, we obtained highly enriched functional terms such as regulation of steroid genesis, orphan nuclear receptors, nerve growth factor, DNA damage, signal transduction, and membrane associated terms. In the present understanding of negative effects of cigarette smoking on humans, the enriched terms and related genes are known to be associated with either cancer progression or nervous system. On the other hand, in case of FSvsNS, the enriched biological terms are generally associated with inflammatory response, extracellular regions, disulfide bonding. As expected, there are not such harmful effects observed in former smoker when compared to never smokers. The interesting observation about this list is that some of these genes such as ADAMS14, SLC38A3, HSD11B1 accommodate structure variation (SNPs) due to tobacco smoking exposure for longer period of time frame.

\section{Additional files}

Additional file 1: Gene-disease-chemical study of Extra-Trees signature in SvsNCS. Gene-disease-chemical association studies for gene signature predicted by Extra-Trees method for smokers versus non-current smokers case study. (CSV $59 \mathrm{~kb}$ )

Additional file 2: Gene-disease-chemical study of LASSO-LARS signature in SvsNCS. Gene-disease-chemical association studies for gene signature predicted by LASSO-LARS method for smokers versus non-current smokers case study. (CSV $30 \mathrm{~kb}$ )

Additional file 3: Gene-disease-chemical study of RFE-SVM signature in SvsNCS. Gene-disease-chemical association studies for gene signature predicted by RFE-SVM method for smokers versus non-current smokers case study. (CSV $50 \mathrm{~kb}$ )

Additional file 4: Gene-disease-chemical of Extra-Trees signature in FSvsNS. Gene-disease-chemical association studies for gene signature predicted by Extra-Trees method for former smokers versus never smokers case study. (CSV $44 \mathrm{~kb}$ )

Additional file 5: Gene-disease-chemical of LASSO-LARS signature in FSvsNS. Gene-disease-chemical association studies for gene signature predicted by LASSO-LARS method for former smokers versus never smokers case study. (CSV $15 \mathrm{~kb}$ )

Additional file 6: Gene-disease-chemical of RFE-SVM signature in FSvsNS. Gene-disease-chemical association studies for gene signature predicted by RFE-SVM method for former smokers versus never smokers case study. (CSV $32 \mathrm{~kb}$ )

Additional file 7: GO-enrichment of common gene signature. Gene ontology enrichment analysis for 8 genes in common with other participants of the SysTox Computational Challenge. (CSV $11 \mathrm{~kb}$ )

Additional file 8: GO-enrichment of specific gene signature. Gene ontology enrichment analysis for 6 genes not in common with other participants of the SysTox Computational Challenge. (CSV 9 kb)

Additional file 9: Pathways-enrichment of common gene signature. Pathways enrichment analysis for 8 genes in common with other participants of the SysTox Computational Challenge. (CSV 2 kb) 
Additional file 10: Pathways-enrichment of specific gene signature. Pathways enrichment analysis for 6 genes not in common with other participants of the sbv IMPROVER SysTox Computational Challenge. (CSV $2 \mathrm{~kb}$ )

Additional file 11: Pathways mapping of common versus specific gene signature. Mapping pathways enrichment for both common and specific gene signature with respect to the complete pathways set associated with tobacco smoke pollution. (CSV 93 kb)

\section{Abbreviations}

AUPR: Area under precision-recall curve; ERT: Extremely randomized trees; FS: Former smokers; FSvsNS: Former smokers versus never smokers; GNB: Gaussian Naive Bayes; GTB: Gradient tree boosting; kNN: k-nearest neighbors; KPT: Kumar Parijat Tripathi (author); LARS: Least angle regression; LASSO: Least absolute shrinkage and selection operator; LASSO-LARS: LASSO with LARS; LDA: Linear discriminant analysis; LR: Logistic regression; MCC: Matthews correlation coefficients; MG: Maurizio Giordano (author); MLP: Multilayer perceptron; MRG: Mario Rosario Guarracino (author); NS: Never smokers; PMI: Philip morris international; QASMC: Queen ann street medical center; RF: Random forests; RFE-SVM: Recursive feature elimination with support vector machines; S: Smokers; sbv IMPROVER: Systems biology verification combined with industrial methodology for process verification in research; SVC: Support vector classifier; SVM: Support vector machine; SvsNCS: Smokers versus non-current smokers; SysTox: Systems toxicology

\section{Acknowledgements}

We would like to thank Giuseppe Jurman from FBK (Trento, Italy) with whom we discussed some ideas that were important to develop our methodology. We would like to acknowledge the efforts of Mr Giuseppe Trerotola from ICAR-CNR (Naples, Italy) for the technical support. Last, but not least, we would like to thank all organizers and participants of the sbv IMPROVER SysTox Computational Challenge: the former because they gave us the possibility to investigate our machine learning methodologies and competences on a very interesting application domain, as well as to use the challenge datasets; the latter because their published results in the SysTox challenge were useful in the comparative study and validation of our results.

\section{Funding}

The activities of this work as well as the publication of this article were sponsored by the project "Laboratory for Genomics, Proteomics and Transcriptomics (LAB-GTP)" funded by Italian Ministry for Universities and Research (MIUR). MRG's work has been conducted at National Research University Higher School of Economics and supported by RSF grant 14-41-00039.

\section{Availability of data and materials}

Transcriptomics datasets used as an assessment and validation of the proposed gene signature selection methodology are not freely available as they are owned by the sbv IMPROVER project website. Their use was allowed only to participants of the SysTox Computational Challenge under the restriction of the sbv IMPROVER Terms of Use. The basic techniques employed and merged in our gene selection methodology, as well as the classification techniques used in the experiments, are software solutions freely available for download and included in the Scikit-learn and Weka Data Mining software, respectively under the GNU and BSD license. All figures and tables included in this document were produced by authors, and they are neither reproduction nor adaptation of existing materials.

\section{About this supplement}

This article has been published as part of BMC Bioinformatics Volume 19 Supplement 2, 2018: Proceedings of Bringing Maths to Life (BMTL) 2017. The full contents of the supplement are available online at https:// bmcbioinformatics.biomedcentral.com/articles/supplements/volume-19supplement-2.

\section{Authors' contributions}

MG and MRG conceived the gene signature selection methodology. MG developed the selection methods and performed all experiments by using off-the-shelf classification techniques. All authors performed the analyses of results: in particular, MRG and KPT carried out the biological and toxicogenomic interpretation of gene signatures, while MG performed the performance analysis of classification methods based on signatures. All authors wrote the manuscript. All authors read and approved the final manuscript.

\section{Ethics approval and consent to participate}

Datasets used in the current work are all from secondary sources, where primary ethics approval had been obtained for data acquisition. Implementations of algorithms and mathematical methods used in the current work are all available as opensource software.

\section{Consent for publication}

Not applicable.

\section{Competing interests}

The authors declare that they have no competing interests.

\section{Publisher's Note}

Springer Nature remains neutral with regard to jurisdictional claims in published maps and institutional affiliations.

\section{Published: 8 March 2018}

\section{References}

1. Christians U, Schmitz V, Schoning W, Bendrick-Peart J, Klawitter J, Haschke M, Klawitter J. Toxicodynamic therapeutic drug monitoring of immunosuppressants: promises, reality, and challenges. Ther Drug Monit. 2008;30(2):151-8. https://doi.org/10.1097/FTD.0b013e31816b9063.

2. Wang E-J, Snyder RD, Fielden MR, Smith RJ, Gu Y-Z. Validation of putative genomic biomarkers of nephrotoxicity in rats. Toxicology. 2008;246(2):91-100. https://doi.org/10.1016/j.tox.2007.12.031.

3. Khor TO, Ibrahim S, Kong A-NT. Toxicogenomics in drug discovery and drug development: Potential applications and future challenges. Pharm Res. 2006;23(8):1659-64. https://doi.org/10.1007/s11095-006-9003-8.

4. Ridd K, Zhang S-D, Edwards RE, Davies R, Greaves P, Wolfreys A, Smith AG, Gant TW. Association of gene expression with sequential proliferation, differentiation and tumor formation in murine skin. Carcinogenesis. 2006;27(8):1556. https://doi.org/10.1093/carcin/bgl007.

5. Martin F, Talikka M, Hoeng J, Peitsch M. Identification of gene expression signature for cigarette smoke exposure response-from man to mouse. Hum Exp Toxicol. 2015;34(12):1200-11. https://doi.org/10.1177/ 0960327115600364 . PMID: 26614807.

6. Tarca AL, Gong X, Romero R, Yang W, Duan Z, Yang H, Zhang C, Wang $P$. Human blood gene signature as a marker for smoking exposure: Computational approaches of the top ranked teams in the sbv improver systems toxicology challenge. Comput Toxicol. 2017. In Press.

7. Hsieh SJ, Ware LB, Eisner MD, Yu L, Jacob III P, Havel C, Goniewicz ML, Matthay MA, Benowitz NL, Calfee CS. Biomarkers increase detection of active smoking and secondhand smoke exposure in critically ill patients. Crit Care Med. 2011;39(1):40-5.

8. Lettieri T. Recent applications of dna microarray technology to toxicology and ecotoxicology. Environ Health Perspect. 2006;114(1):4-9. https://doi. org/10.1289/ehp.8194.

9. Rempel E, et al. A transcriptome-based classifier to identify developmental toxicants by stem cell testing: Design, validation and optimization for histone deacetylase inhibitors. Arch Toxicol. 2017;89(9):1599-618. https:// doi.org/10.1007/s00204-015-1573-y.

10. Pallocca G, Grinberg M, Henry M, Frickey T, Hengstler JG, Waldmann T, Sachinidis A, Rahnenführer J, Leist M. Identification of transcriptome signatures and biomarkers specific for potential developmental toxicants inhibiting human neural crest cell migration. Arch Toxicol. 2016;90(1): 159-80. https://doi.org/10.1007/s00204-015-1658-7.

11. De Gregorio M, Giordano M. Cloning DRASiW systems via memory transfer. Neurocomputing. 2016;192:115-27. https://doi.org/10.1016/j. neucom.2016.01.087.

12. Viola M, Sangiovanni M, Toraldo G, Guarracino MR. A generalized eigenvalues classifier with embedded feature selection. Optimization Letters. 2017;11(2):299-311. https://doi.org/10.1007/s11590-015-0955-7.

13. Guarracino MR, Cuciniello S, Pardalos PM. Classification and characterization of gene expression data with generalized eigenvalues. J Optim Theory Appl. 2009;141(3):533-45. https://doi.org/10.1007/ s10957-008-9496-x. 
14. sbv IMPROVER / System Biology Verification. http://sbvimprover.com. Accessed 12 Oct 2017.

15. Belcastro V, Poussin C, Xiang Y, Giordano M, Tripathi KP, Boda A, Balci AT, Bilgen I, Dhanda SK, Duan Z, Gong X, Kumar R, Romero R, Sarac OS, Tarca AL, Wang P, Yang H, Yang W, Zhang C, Boué S, Guarracino MR, Martin F, Peitsch MC, Hoeng J. The sbv improver systems toxicology computational challenge: Identification of human and species-independent blood response markers as predictors of smoking exposure and cessation status. Comput Toxic. 2017. https://doi. org/10.1016/j.comtox.2017.07.004

16. SysTox Challenge | sbv IMPROVER. http://sbvimprover.com/systoxchallenge. Accessed 12 Oct 2017.

17. Titz B, Sewer A, Schneider T, Elamin A, Martin F, Dijon S, Luettich $K$, Guedj E, Vuillaume G, Ivanov NV, Peck MJ, Chaudhary NI, Hoeng J, Peitsch MC. Alterations in the sputum proteome and transcriptome in smokers and early-stage \{COPD\} subjects. J Proteome. 2015;128:306-20. https://doi.org/10.1016/j.jprot.2015.08.009.

18. ArrayExpress < EMBL-EBI. http://www.ebi.ac.uk/arrayexpress. Accessed 12 Oct 2017.

19. Geurts P, Ernst $D$, Wehenkel L. Extremely randomized trees. Mach Learn. 2006;63(1):3-42. https://doi.org/10.1007/s10994-006-6226-1.

20. Efron B, Hastie T, Johnstone I, Tibshirani R. Least angle regression. Ann Stat. 2004;32(2):407-51.

21. Guyon I, Weston J, Barnhill S, Vapnik V. Gene selection for cancer classification using support vector machines. Mach Lear. 2002;46: 389-422. https://doi.org/10.1023/A:1012487302797.

22. Pedregosa F, Varoquaux G, Gramfort A, Michel V, Thirion B, Grisel O, Blondel M, Prettenhofer P, Weiss R, Dubourg V, Vanderplas J, Passos A, Cournapeau D, Brucher M, Perrot M, Duchesnay E. Scikit-learn: Machine learning in python. J Mach Learn Res. 2011;12:2825-30.

23. Hall $M$, Frank $E$, Holmes $G$, Pfahringer $B$, Reutemann $P$, Witten $I H$. The weka data mining software: An update. SIGKDD Explor Newsl. 2009;1 1(1): 10-8. https://doi.org/10.1145/1656274.1656278.

24. Davis AP, Grondin CJ, Johnson RJ, Sciaky D, King BL, McMorran R, Wiegers J, Wiegers TC, Mattingly CJ. The comparative toxicogenomics database: update 2017. Nucleic Acids Res. 2017;45(D1):972-8. https://doi. org/10.1093/nar/gkw838.

25. Tripathi KP, Evangelista D, Zuccaro A, Guarracino MR. Transcriptator: An automated computational pipeline to annotate assembled reads and identify non coding rna. PLOS ONE. 2015;10(11):1-17. https://doi.org/10. 1371/journal.pone.0140268.

26. Beineke P, Fitch $K$, Tao H, Elashoff MR, Rosenberg S, Kraus WE, Wingrove JA. A whole blood gene expression-based signature for smoking status. BMC Med Genomics. 2012;5(1):58. https://doi.org/10. 1186/1755-8794-5-58.

27. Poussin C, Belcastro V, Martin F, Boué S, Peitsch MC, Hoeng J. Crowd-sourced verification of computational methods and data in systems toxicology: A case study with a heat-not-burn candidate modified risk tobacco product. Chem Res Toxicol. 2017;30(4):934-45. https://doi.org/10.1021/acs.chemrestox.6b00345.

\section{Submit your next manuscript to BioMed Central and we will help you at every step:}

- We accept pre-submission inquiries

- Our selector tool helps you to find the most relevant journal

- We provide round the clock customer support

- Convenient online submission

- Thorough peer review

- Inclusion in PubMed and all major indexing services

- Maximum visibility for your research

Submit your manuscript at www.biomedcentral.com/submit

C Biomed Central 